The British Journal of Nursing Studies

DOI: 10.32996/bjns

Journal Homepage: www.al-kindipublisher.com/index.php/bjns

\title{
Predicting Big Five Model Personality Traits in Recent Social Context
}

\author{
Md. Mahfuzur Rahman Khan \\ Institute of Social Welfare \& Research, University of Dhaka, Bangladesh
}

$\square$ Corresponding Author: Md. Mahfuzur Rahman Khan, E-mail: mahfuzur.rkm@gmail.com

\begin{abstract}
ARTICLE INFORMATION
Received: 08 October 2021

Accepted: 14 November 2021

Published: 23 November 2021

DOI: 10.32996/bjns.2021.1.1.1

\section{KEYWORDS}

Big Five Model, Personality, Personality Traits, Social Context.

\section{ABSTRACT}

The theory of the five great factors of personality (Big Five theory) is currently considered to be the best at explaining the most individual variability in terms of personality factors between subjects. The purpose of this study is to examine the relationship between the Big Five personality traits and the recent social context, and to determine whether social support can act as a moderating factor in the process. While previous research has established that personality traits are significant predictors of well-being, the extent to which the big five personality traits influence social well-being remains unknown. This research paper employs a qualitative method based on a deductive research approach, as the author used deductive logic to predict the big five model personality traits in a recent social context. Five personality traits were found to be significantly related to the overall social context and social support functions as a determinant in the relationships between extraversion/agreeableness/conscientiousness/neuroticism/openness and social context. Numerous studies now concur, and there is a high degree of consensus in the literature regarding the Big Five theory as a useful theory for identifying and describing the major general factors of personality.
\end{abstract}

\section{Introduction}

Several personality instructive models place a premium on the social environment. Personality traits and social context are presented as two themes that are investigated in a variety of studies, with a focus on those that investigate the relationship between (indicators of both. The majority of these studies are based on the model of the five great personality factors. As a result, these studies investigated the theoretical and empirical relationships between personality dimensions defined by the Big Five Factor model and social context. These variables are conceptually and empirically related, according to the findings. As a result, personality and social context may influence one another. Given that social context is offered as a tool, effective integration into the social context is important, and the environment plays a role in the development of various characteristics displayed by the person, the goal of this article is to investigate the relationship between personality traits and components of social context, using the big five model of personality traits. Society has specific expectations of us, which it communicates to us via the models it imposes on its members. Each, in fact, refers to a prototype to define the characteristics of the ideal person that are admired and those that are despised. In terms of personality, on the other hand, the unique characteristics that distinguish each individual can be highlighted, while recognising similarities with other individuals. An individual's character is strongly influenced by the environment during his childhood and adolescence, and is thus linked to the history presented and cultural heritage learned during development. The structure of the personality refers to how the personality presents itself and manifests itself in personality traits. The trait is characterised by a proclivity to act relatively independently of changing situations and contexts, or by a proclivity to experience and regulate emotions and affects, process information, and act in a relatively uniform manner. Various theories and authors have attempted to explain personality through traits, with differing viewpoints on the nature and origin of traits, as well as their number. The dynamics of personality, on the other hand, is concerned with the functioning of the personality as a system capable of reflecting on itself and interacting with its environment in order to achieve specific goals. Thus, the dynamics of the personality refer to the processes and mechanisms that preside over the construction of identity, which regulate behaviours and allow the individual to adapt and satisfy their needs, which support dispositions and strategies that provide continuity and stability to the individual experience. Personality is a psychology construct that has always stood out in the field of Psychological Assessment, which has led to a plethora of theoretical and methodological studies and debates. The authors attribute the model's significance to the fact that it has been applied in various samples, cultures, and through numerous sources of information (including self-

Copyright: (C) 2021 the Author(s). This article is an open access article distributed under the terms and conditions of the Creative Commons Attribution (CC-BY) 4.0 license (https://creativecommons.org/licenses/by/4.0/). Published by Al-Kindi Centre for Research and Development, London, United Kingdom. 
assessment, peer review, and clinical assessments), demonstrating its suitability for various uses. Although there are several definitions for this concept, the personality assessment will be determined by the theory used by the researcher, so the way theories conceptualize the term ends up defining the main characteristics of each theoretical position. The Big Five Personality Factor model, also known as the Big Five, is considered an explanatory and predictive theory of personality. It is one of the most widely used models to describe the personality structure within trait theory, particularly adult personality from a psychometric standpoint. Individuals can benefit from social support to help them avoid the negative health consequences of life stress and to improve their overall well-being (Cobb, 1976; Siedlecki et al., 2014). Thus, the current study examined not only the relationship between five personality traits and recent social context, but also whether social support can act as a moderator in the relationship between the big five personality traits and social context.

\section{Literature Review}

Psychologists having expertise in personality are primarily concerned with "providing an integrative framework for comprehending the whole person" (McAdams \& Pals, 2006). In recent years, the "Big Five" personality traits have emerged as the dominant framework for measuring personality traits in psychology. Psychologists classify these characteristics as dispositional or fundamental. This label differentiates the Big Five from other facets of an individual's personality, such as their distinctive adaptations (values, attitudes, in criteria), self-concepts (self-esteem, identity), and objective biography (McAdams \& Pals, 2006; Costa \& Paul, 1996). Unlike other aspects of personality that develop and change over the course of a person's life, dispositional traits are viewed as stable characteristics of individuals that dictate how they respond to the diverse stimuli they encounter in the world. As such, they exert a wide range of influence over behaviours and attitudes in a variety of situations. According to McCrae and Costa (2008), personality trait research is predicated on four assumptions about human nature: (a) personality traits exist and are quantifiable; (b) these traits vary between individuals. (c) the causes of human behaviour are intrinsic to the individual (personality traits, for example, influence individual behaviour), and (d) people "can comprehend themselves and others." Numerous authors and addresses in the field of personality psychology have addressed the same in terms of its structural aspects, that is, the architecture of dispositions. These guidelines are predicated on the premise that psychological behaviours and manifestations are explained by a latent structure of traits or fundamental dispositions. Individuals have complex personalities. Consider personality in terms of just five factors. This may make it appear to be a straightforward matter. However, it should be noted that the Big Five encompass a broad range of personality traits that have historically and across cultures aided people in establishing relationships within their own social groups and progressing up the social hierarchy.Although there are numerous archetypes of personality traits, researchers have agreed on the Five-Factor or Big Five model as a way to describe the most salient aspects of personality traits (Barrick, 2005). The Big Five, as well as other models that specify human personality traits, are derived from Francis Galton's nineteenth-century lexical hypothesis. The Big Five trait domains were identified through a thorough lexical analysis of each natural language. The lexical hypothesis states that each natural language contains all personality descriptions that are relevant and significant to its speakers. Gordon Allport and his colleague Henry Odbert investigated this hypothesis in 1936 by poring over a comprehensive English dictionary and compiling a list of 18,000 terms referring to individual differences. Approximately 4,500 of these terms referred to personality characteristics. This vast set of terms provided a starting point for psychologists interested in the lexical hypothesis, but it was unsuitable for research, and thus other scholars attempted to narrow the word set. This approach is predicated primarily on the fourth assumption: that people understand one another and that others understand, and that a significant historical function of language was to enable people to describe the persistent differences between individuals (Allport \& Odbert, 1936; John et al., 2008). Thus, languages have evolved over time to include terms that facilitate the identification of the most salient and pervasive individual differences among people. The Big Five, on the other hand, did not receive widespread scholarly attention until the 1980s. Today, the Big Five are an integral part of psychological research, and psychologists generally agree that personality can be classified into the Big Five's five basic traits. Each segment of the Big Five is a point on a continuum. Introversion, for example, is the polar opposite of extraversion. Extraversion and introversion are polar opposites on the Big Five trait spectrum. Individuals can be extremely outgoing or extremely introverted, but the majority of people fall somewhere in between these two extremes. Additionally, it is critical to remember that each of the Big Five traits is extremely broad and encompasses a variety of personality characteristics. These characteristics are more precise and nuanced than the five traits individually. As a result, each trait can be defined broadly and also subdivided into distinct facets. Gordon Allport (1927) distinguished traits as fundamental units of personality into individual, common, cardinal, central, and secondary traits. Different types of traits influence behaviour and distinguish individuals and personalities to varying degrees. Personality is defined as the sum of an individual's characteristics that can be used to explain observed behaviour. Thus, traits are latent (i.e. not directly observable) variables that contribute to the explanation of observed human behaviour. Cattell (1943) identified sixteen primary personality characteristics, including the following: A-detached, cold; B-superficial, intelligent; and C-immature, labile.

\section{Methodology}

This research paper employs a qualitative method based on a deductive research approach, as the author used deductive logic to predict the big five model personality traits in a recent social context. Because deductive reasoning is used to progress from the particular to the general. If a causal relationship or link appears to be implied by a particular theory or case example, it may be true in many cases, and deductive design may be used to determine whether this relationship or link did exist under more general 
conditions. Using a deductive approach, individuals develop a hypothesis (or hypothesises) based on current theory and then design an investigation strategy to test the hypothesis (Wilson, 2014).

\section{Recent Social Context and the Big Five Personality Traits}

Personality is complicated and variable, and each individual may exhibit actions that span several of these characteristics at the same time. Art, emotion, adventure, novel ideas, imagination, curiosity, and a wide range of experiences are all appreciated by those who are open to new things and are willing to learn. People who are open to experience are intellectually curious, open to emotion, sensitive to beauty, and willing to try new things. They are also open to new ideas and new experiences. If you compare open people to closed ones, you will find that they are more creative and more sensitive to their sentiments. They are also more likely to have beliefs that are out of the ordinary. High openness can be interpreted as unpredictability or a lack of focus, and it might lead to an increased likelihood of engaging in dangerous conduct or drug use. Conscientiousness is a personality attribute that refers to being cautious or diligent in one's actions. Conscientiousness is defined as the desire to perform a task well and to take one's responsibilities to others seriously. People who are conscientious tend to be efficient and ordered, as opposed to those who are easy-going and untidy. They have a strong tendency to exercise self-discipline, carry out their responsibilities, and strive for success. They engage in planned rather than spontaneous activities, and they are generally dependable. It manifests itself in distinctive behaviours such as being orderly and methodical, as well as in components such as carefulness, thoroughness, and deliberation, among other characteristics (the tendency to think carefully before acting). Extraversion (sometimes written extroversion) and introversion (also spelled extroversion) are two characteristics that are essential to several theories of human psychology. When it comes to conduct, extraversion is typically manifested as extroverted, talkative, and energetic, whereas introversion is typically manifested as more reflective and reserved behaviour. Typically, the traits of extraversion and introversion are considered as a single continuum, so that to be high in one requires that one be low in the other. Extraverts are stimulated and thrive when they are in the company of other people. It is enjoyable for them to participate in events that include huge social gatherings such as birthday celebrations, neighbourhood gatherings, public demonstrations, and meetings of commercial or political organisations. They also have a proclivity to work well in groups. When it comes to time spent with other people, an extraverted person is more likely to find it rewarding than when spending time alone. They are more stimulated when they are in the company of other people, and they are more prone to boredom when they are alone in their thoughts. Acceptability is a personality attribute that manifests itself in individual behavioural characteristics that are viewed as kind, sympathetic, cooperative, warm, and considerate by those who observe them. Individual differences in cooperation and social harmony are reflected in individual differences in agreeableness, which is one of the five major aspects of personality structure in contemporary personality psychology. People who have a high agreeableness score are empathic and altruistic, whereas those who have a low agreeableness score engage in selfish behaviour and show little empathy for others. Those who score extremely low on agreeableness exhibit evidence of dark triad behaviour, such as manipulating and competing with others rather than cooperating, according to the study. When it comes to personality traits, agreeableness is considered a superordinate trait, which means that it is a collection of subtraits that statistically cluster together. Trust, straightforwardness, altruism, obedience, modesty, and tender-heartedness are some of the lower-level characteristics or qualities that fall under the umbrella term "agreeableness." Neuroticism is a personality trait that appears in various models of personality theory, but there is great disagreement about how it should be defined. If you are aroused, you have a propensity to quickly become alert and then slowly relax after becoming alert. This is especially true if you are exposed to unpleasant emotional arousal. Yet another definition emphasises the difference between emotional instability and negativity, as well as maladjustment, as opposed to emotional stability and positivity, as well as successful adjustment. It has also been defined in terms of a lack of self-control, a poor ability to cope with psychological stress, and a proclivity to complain, among other characteristics. Individuals with low neuroticism tend to be more emotionally stable and less reactive to stress than those with high neuroticism. They have a tendency to be calm and even-tempered, and they are less likely to become uptight or agitated. Despite the fact that they have low levels of negative emotions, they do not necessarily have large levels of pleasant feelings. Extraversion is an autonomous characteristic that is characterised by high levels of positive emotion. Being high in positive emotion scores is typically an element of extraversion. Extraverts with neurotic tendencies, for example, might experience high levels of both good and negative emotional states at the same time, creating a "emotional roller coaster." Extraversion, neuroticism, openness, agreeableness, and conscientiousness comprise the big five personality traits (John and Srivastava, 1999). Extraversion is a term that refers to the degree to which an individual is vivacious, sociable, talkative, and gregarious. Agreeableness is a measure of how warm, caring, supportive, and cooperative an individual is, as well as how well they get along with others. Conscientiousness refers to the degree to which an individual is well-organized, accountable, punctual, goal-oriented, and dependable. Neuroticism refers to a person's level of worry, anxiety, impulsivity, and insecurity. Openness reflects one's capacity for imagination, creativity, curiosity, and open-mindedness (Barrick et al., 2001; Funder and Fast, 2010). Numerous scholars have used a combined eidetic approach to assess personality in various cultural contexts (John and Srivastava, 1999; Cheung et al., 2001). Social support is consistently associated with personality traits (Swickert et al., 2010; Udayar et al., 2018), and social support is associated with the big five personality characteristics. Neurotic individuals report being more susceptible to stress and negative affectivity, which may limit their access to social support (Ayub, 2015). Individuals who score highly on extraversion are constantly on the lookout for social interactions and are generally cheerful and friendly. Positive emotions may result in an increase in social support for the 
individual (Swickert et al., 2010). Individuals who are highly receptive to experience demonstrate increased emotional receptivity, an appreciation for art and beauty, intellect, and liberalism. These characteristics would suggest a higher likelihood of receiving social support (Barańczuk, 2019). Individuals who exhibit characteristics of agreeableness such as modesty, compliance, and trust may be able to expand their social support network (Barańczuk, 2019). Conscientiousness is defined by a desire for accomplishment, self-discipline, orderliness, and responsibility. These characteristics can help individuals cope with life stress, which is why they are positively associated with social support (Ayub, 2015).

\section{Results and Discussion}

Numerous studies now concur, and there is a high degree of consensus in the literature regarding the Big Five theory as a useful theory for identifying and describing the major general factors of personality. As mentioned previously, the theory of the five great factors of personality (Big Five theory) is currently considered to be the best at explaining the most individual variability in terms of personality factors between subjects. Goldberg (1981) coined the term "Big Five," although Norman (1963) was the first to conduct in-depth research on the subject. McCrae and Costa's theory is one of the most widely shared and empirically tested among models focusing on a homothetic approach to the study of personality. If psychoanalysts agree on a general typology of personalities, it is exceedingly difficult to fully comprehend someone's personality in a short period of time. Thus, even without being a specialist, we can deduce that obsessive individuals generally react in some way. From there, there is a step that some people skip when determining whether someone is obsessive. It does, however, invite us to re-read situations with a more benign eye, rather than simply looking for irrationality or inconsistency. There are frequently very compelling reasons, but unconscious ones, for someone to react one way or another. Recognizing that this is possible is a good first step toward higher-quality questioning. While personality is partly determined by biology, its development and differentiation are influenced by context, which has a significant impact on the development of an individual's self-regulatory context in particular. These circumstances enable him to conform to social norms. Since the 1990s, considerable research has concentrated on the universality of various personality models. The majority of studies have demonstrated that these models are capable of accurately describing the personalities of individuals from a wide variety of cultures. As a result, they appear to be universal, even if certain characteristics are culturally specific. Additionally, this research demonstrates how difficult it is to compare the expression of personality behaviours across cultures, because the cultural context has a significant influence on this expression; most personality theories believe that the cultural context has an immediate and rapid effect on the behavioural expression of the personality via its action on the individual's self-regulatory processes. Context, on the other hand, has a more gradual effect on the personality, influencing its development over time. Individuals develop a self-image or personal identity, which includes a representation of themselves and their usual way of being, depending on their personality as well as their social and cultural context. Additionally, individuals have an effect on their surroundings and thus contribute to their development. Additionally, personality has an effect on how we perceive our environment. Thus, an individual who is emotionally unstable, sensitive to stress, unconscientious, and disorganised will perceive his environment as more stressful, for example. Adulthood is characterised by a high degree of stability in personality traits. While gradual changes in personality traits are possible, they are rarely drastic. In other words, if an individual lacks an extrovert trait (i.e., he is more introverted than extroverted), he is likely to remain so, even if his extroversion may fluctuate slightly over time. Additionally, the environment can indirectly reinforce inherited characteristics. For instance, when parents create an environment that complements their own characteristics, they also create an environment that complements their children's characteristics. Similarly, as adults, individuals seek out environments that reinforce and support their characteristics. The Five Factors Model identifies dispositional trends that can be precisely described through the use of a set of five dimensions. According to proponents of the Five Factors Model, everything that occurs outside is due to dispositional trends, which are explained by a set of five phenotypic dimensions of an organisation that describe individual differences. The Five Factors are capable of providing concepts through which people's characteristics can be abstracted (De Raad, 1998).

\section{Conclusion}

These five factors have a lot of practical value, and it's helped a lot in clarifying how they relate to the various types of evaluation instruments used. For professionals who need to simplify analysis to better evaluate individual differences, it summarises measures of those differences (Caprara et al., 1994). Because a small number of factors can accurately represent a large number of observable behavioural variations, dispositional studies have typically used factor analysis. A factor can be seen as a kind of psychological structure that controls how well the personality works. In other words, factor analysis can provide useful information about human nature, but the mechanisms that underlie these insights remain a mystery. The Big Five Personality Factors model was the subject of two surveys. We set out to do two things: first, analyse articles about this model that were found in two well-known electronic databases, Scielo and Pepsic. Choosing databases was based on how important they are for researchers in psychology and related fields to have easy access to scientific production thanks to electronic publication of periodicals and free internet availability. A second survey of the findings can be conducted to add to the work already done in periodic databases. Because of this, despite the fact that studies involving the elderly and children were virtually nonexistent, researchers were able to establish the validity of its use in any society in various samples and with various instruments. Because most instruments have been validated for use with adolescents and adults, and because there is currently no specific instrument for children, this is understandable. To define and 
quantify the influence of various intermediate variables, future research can advance hypotheses based on the model and conduct empirical analysis to demonstrate the model's utility in practical applications (Khan, 2020). It highlights future research needs, such as the development of research involving sample expansion, focusing on elderly and child personalities, and evaluating the model's validity at different stages of a person's life, among other things.. Personality assessment will be able to grow and improve in thanks to advances in research, particularly those aimed at validating instruments based on this model.

Conflicts of Interest: The author declares that there is no conflict of interest.

\section{References}

[1] Allport, G. W. (1927). Concepts of trait and personality. Psychological Bulletin, 24(5), 284.

[2] Allport, G. W., \& Odbert, H. S. (1936). Trait-names: A psycho-lexical study. Psychological monographs, 47(1), i.

[3] Ayub, N. (2015). Predicting suicide ideation through intrapersonal and interpersonal factors: The interplay of Big-Five personality traits and social support. Personality and mental health, 9(4), 308-318.

[4] Barańczuk, U. (2019). The Five Factor Model of personality and social support: A meta-analysis. Journal of Research in Personality, 81, 38-46.

[5] Barrick, M. R. (2005). Yes, personality matters: Moving on to more important matters. Human performance, 18(4), 359-372.

[6] Barrick, M. R., Mount, M. K., \& Judge, T. A. (2001). Personality and performance at the beginning of the new millennium: What do we know and where do we go next?. International Journal of Selection and assessment, 9(1-2), 9-30.

[7] Caprara, G. V., Barbaranelli, C., \& Livi, S. (1994). Mapping personality dimensions in the Big Five model. European Review of Applied Psychology, 44(1), 9-15.

[8] Cattell, R. B. (1943). The description of personality: Basic traits resolved into clusters. The journal of abnormal and social psychology, 38(4), 476.

[9] Cheung, F. M., Leung, K., Zhang, J. X., Sun, H. F., Gan, Y. Q., Song, W. Z., \& Xie, D. (2001). Indigenous Chinese personality constructs: Is the five-factor model complete?. Journal of cross-cultural psychology, 32(4), 407-433.

[10] Costa, J. R., \& Paul, T. (1996). Of personality theories: Theoretical contexts for the five-factor model. The five-factor model of personality: Theoretical perspectives, 51

[11] De Raad, B. (1998). Five big, big five issues: Rationale, content, structure, status, and crosscultural assessment. European Psychologist, 3(2), 113-124.

[12] Funder, D. C., \& Fast, L. A. (2010). Personality in social psychology.

[13] Goldberg, L. R. (1981). The big five personality dimensions.

[14] John, O. P., Naumann, L. P., \& Soto, C. J. (2008). Paradigm shift to the integrative Big Five trait taxonomy: History, measurement, and conceptual issues.

[15] John, O. P., \& Srivastava, S. (1999). The Big-Five trait taxonomy: History, measurement, and theoretical perspectives (Vol. 2, pp. 102-138). Berkeley: University of California.

[16] Khan, M. M. R. (2020). Strategic human resource management in facilitating the organizational performance: Birds-eye view from Bangladesh. Annals of Management and Organization Research, 2(1), 13-24.

[17] McAdams, D. P., \& Pals, J. L. (2006). A new Big Five: fundamental principles for an integrative science of personality. American psychologist, 61(3), 204.

[18] McCrae, R. R., \& Costa Jr, P. T. (2008). The five-factor theory of personality.

[19] Norman, W. T. (1963). Toward an adequate taxonomy of personality attributes: Replicated factor structure in peer nomination personality ratings. The journal of abnormal and social psychology, 66(6), 574.

[20] Swickert, R. J., Hittner, J. B., \& Foster, A. (2010). Big Five traits interact to predict perceived social support. Personality and Individual Differences, 48(6), 736-741.

[21] Udayar, S., Urbanaviciute, I., \& Rossier, J. (2020). Perceived social support and Big Five personality traits in middle adulthood: A 4-year crosslagged path analysis. Applied Research in Quality of Life, 15(2), 395-414.

[22] Wilson, J. (2014). Essentials of business research: A guide to doing your research project. Sage 